Cite this: Phys. Chem. Chem. Phys.,

Received 6th January 2015 Accepted 26th March 2015

DOI: $10.1039 / c 5 c p 00071 h$

www.rsc.org/pccp

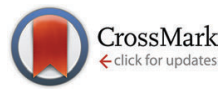
2015, 17, 11647

\section{First principles investigation of the activity of thin film Pt, Pd and Au surface alloys for oxygen reduction $\dagger$}

\author{
Vladimir Tripkovic, ${ }^{\text {abc }}$ Heine Anton Hansen, ${ }^{a}$ Jan Rossmeisl ${ }^{b c}$ and Tejs Vegge ${ }^{a}$
}

Further advances in fuel cell technologies are hampered by kinetic limitations associated with the sluggish cathodic oxygen reduction reaction. We have investigated a range of different formulations of binary and ternary Pt, Pd and Au thin films as electrocatalysts for oxygen reduction. The most active binary thin films are near-surface alloys of Pt with subsurface Pd and certain PdAu and PtAu thin films with surface and/or subsurface $\mathrm{Au}$. The most active ternary thin films are with pure metal Pt or Pd skins with some degree of $\mathrm{Au}$ in the surface and/or subsurface layer and the near-surface alloys of Au with mixed Pt-Pd skins. The activity of the binary and ternary catalysts is explained through weakening of the $\mathrm{OH}$ binding energy caused by solute elements. However, given the low alloy formation energies it may be difficult to tune and retain the composition under operating conditions. This is particularly challenging for alloys containing $\mathrm{Au}$ due to a high propensity of Au to segregate to the surface. We also show that once Au is on the surface it will diffuse to defect sites, explaining why small amounts of Au retard dissolution of Pt nanoparticles. For the PtPd thin films there is no pronounced driving force for surface segregation, diffusion to defects or surface self-assembling. On the basis of stability and activity analysis we conclude that the near surface alloy of Pd in Pt and some PdAu binary and PtPdAu ternary thin films with a controlled amount of $\mathrm{Au}$ are the best catalysts for oxygen reduction.

\section{Introduction}

The development of new materials in the field of energy conversion and storage is a prerequisite for finding an alternative to the environmentally detrimental use of fossil fuels. One possible solution is to use the proton exchange membrane fuel cell (PEMFC). However, the PEMFC technology is not cost-competitive with contemporary internal combustion engines due to the high cost of the platinum catalyst. Reducing the Pt-loading at the cathode by improving the catalyst performance (activity \& stability) is a key factor needed for commercialization of the technology. However, the Pt loading cannot be reduced without overcoming kinetic limitations associated with the cathodic oxygen reduction reaction (ORR) and/or extending the life-time of the catalyst., Although, the maximum theoretical efficiency in PEMFCs is $83 \%$, in practice, the efficiency is about half of the theoretical value. The loss of catalytic activity is mainly attributed to surface

\footnotetext{
${ }^{a}$ Department of Energy Conversion and Storage, Technical University of Denmark, DK-4000 Roskilde, Denmark. E-mail: tripce@gmail.com

${ }^{b}$ Center for Atomic-scale Materials Design, Department of Physics, Technical University of Denmark, DK-2800 Kgs. Lyngby, Denmark

Nano-Science Center, Department of Chemistry, University of Copenhagen,

Universitetsparken 5, DK-2100 Copenhagen, Denmark

$\dagger$ Electronic supplementary information (ESI) available. See DOI: 10.1039/c5cp00071h
}

poisoning of the catalyst by water oxidation products. The poisoning shifts the working potential by $c a$. $0.4 \mathrm{~V}$ from the equilibrium potential even on the most active Pt-group catalysts. $^{3-7}$ The stability loss, on the other hand, is associated with platinum dissolution under highly corrosive conditions at the PEMFC cathodes. ${ }^{2}$ Several strategies to improve the activity and stability performance have been implemented over the past decades. For instance, the Pt activity can be satisfactorily tuned by alloying Pt with non-noble metal elements. ${ }^{8-13}$ The idea behind alloying is to change the electronic properties of the platinum surface in order to retard the onset potential for water oxidation. This, in turn, will increase the working potential and the overall efficiency of the cell. Activity enhancements might, however, be offset by degradation of the membrane electrode assembly caused by leaching-out of base-metals and contamination of the ion-exchange sites in the membrane. ${ }^{2}$ Alloying Pt with metals that have higher dissolution potentials, such as noble metals, can overcome this problem.

Different alloys of Pt, Pd and Au have previously been investigated as potential catalysts for oxygen reduction. In the following section we will examine the current status of research on these alloys. The activity for the ORR of Pt(111) skins on late-transition metals has been systematically analyzed in the past. ${ }^{14} \mathrm{~A} \operatorname{Pt}(111)$ monolayer on $\operatorname{Pd}(111)$ stood out as the only catalyst whose activity 
surpassed that of $\mathrm{Pt}(111)$. The same analysis performed for Pd(111) skins showed no activity improvement whatsoever with respect to $\operatorname{Pd}(111) .{ }^{15,16}$ Different formulations of PtPd bulk catalysts have been synthesized and tested for oxygen reduction. In ref. 17, the authors found that non-supported PtPd aerogels exhibited a volcano-type dependence on the Pd content, with the highest activity centered at $20-30 \% \mathrm{Pd}$. The virtue of these catalysts is that they combine high stability of extended surfaces with high surface area of nanoparticulate catalysts. Other nanostructured PtPd alloys have also shown very encouraging results. ${ }^{18-27}$ Despite the fact that Pt and Au are immiscible in the whole concentration range, it is possible to make PtAu alloys using specific synthesis procedures. ${ }^{28-30}$ The activity of PtAu was found to be strongly dependent on the catalyst pretreatment. After heating the catalyst in a CO atmosphere or cycling it into the oxide formation region, the observed activity was greater than that on Pt. ${ }^{26,28,31-34}$ The activity enhancement was attributed to a reversible surface segregation of $\mathrm{Pt}$ induced by $\mathrm{CO}$ or $\mathrm{O}$ adsorbates. Au has a low surface energy and thus naturally prefers to be on the surface. On the other hand, CO binds more strongly to Pt, which can stabilize $\mathrm{Pt}$ in the surface layer. In addition to prompting the activity, Au has shown another beneficial effect on Pt. Small Au clusters added to Pt nanoparticles improved its stability by increasing the average nanoparticle dissolution potential. ${ }^{35,36} \mathrm{PdAu}$ alloys have mainly been used as electrocatalysts for the oxidation of small organic molecules such as $\mathrm{CO},{ }^{37}$ formic acid, ${ }^{38,39}$ ethanol $^{40}$ or glycerol. ${ }^{41}$ The only reported study on the ORR activity was for PdAu nanowires. ${ }^{42}$ The PdAu nanowires displayed an approximately two-fold activity increase with respect to archetypal Pt. As for the ternary catalysts, there are promising new reports for nanoparticles with Pt shell on a Pd core with some additional gold in the subsurface layer. ${ }^{42}$ The role of gold was ascribed to lowering the activity loss during operation.

In this work using density functional theory (DFT) calculations, we systematically investigate different formulations of Pt, Pd and Au binary and ternary thin films for the ORR. We establish composition-activity and activity-stability relationships using the binding energy (BE) of $\mathrm{OH}$ and surface energy of the thin film terminated slab as activity and stability descriptors. Furthermore, we discuss stability in terms of how easily the composition can be changed during operation and the driving forces behind the change. More specifically, we analyze and compare surface segregation energies without the presence of adsorbates and with $\mathrm{OH} / \mathrm{O}$ at a fixed $1 / 3$ monolayer (ML) coverage. In addition, we examine the site and distribution preference for segregation. The site preference indicates whether solute atoms segregate more favorably to terraces or defects. The distribution preference indicates whether solute atoms segregate forming uniform distributions, alike those in surface alloys, or distinct solute enriched domains. For establishing the composition-activity and activitystability relationships, we employ the most stable and welldefined close-packed (111) termination. For the analysis of a defect driven diffusion, we employ the stepped (221) surface.

\section{Results and discussion}

\subsection{Binary alloys}

Six binary combinations of Pt, Pd and Au elements are initially investigated, i.e. Pt@PtPd, Pt@PtAu, Pd@PtPd, Pd@PdAu, Au@PtAu and Au@PdAu, where composition before the @ sign is for the core element and after for the thin films. Utilizing a $\sqrt{ } 3 \times \sqrt{ } 3 R 30^{\circ}$ unit cell size allows the composition in each layer to be varied uniformly in increments of $1 / 3 \mathrm{ML}$, yielding in total 16 possible compositions for the two surface layers. Four possible arrangements of the host and solute elements in the surface layer are presented in Fig. 1. Two out of six thin films, Au@PtAu and $\mathrm{Au} @ \mathrm{PdAu}$ are discarded a priori. Presumably these thin films will not be active for the ORR due to the large Au lattice constant. Overlaying a Au substrate with a Pt or Pd overlayer will induce a tensile strain in the overlayer. This will, in turn, increase the $\mathrm{OH}$ BE making the catalysts even more prone to surface poisoning. As a result, the ORR activity will decrease. ${ }^{11,16}$ Hereafter, in order to distinguish between different compositions, in the text the thin alloy films are denoted by the composition of the surface layer, subsurface layer and bulk using the following nomenclature 1st layer/2nd layer/bulk composition. The composition in the 1st and 2nd layer is denoted by the number of atoms in the surface unit cell, and bulk composition by the host element symbol. For example, the $\mathrm{Pt}_{2} \mathrm{Au} / \mathrm{Pt}_{3} / \mathrm{Pt}$ notation denotes a thin film that has two Pt and $1 \mathrm{Au}$ atom in the surface layer, $3 \mathrm{Pt}$ atoms in the subsurface layer while rest is bulk Pt. We use the same nomenclature in the figures with a difference that the host element is given by color; red for Pt and blue for Pd.

2.1.1. Activity. We assume that the ORR follows an associative reaction pathway, which proceeds through the following sequence of intermediates $\mathrm{O}_{2} \rightarrow \mathrm{OOH} \rightarrow \mathrm{O} \rightarrow \mathrm{OH} \rightarrow \mathrm{H}_{2} \mathrm{O}$.
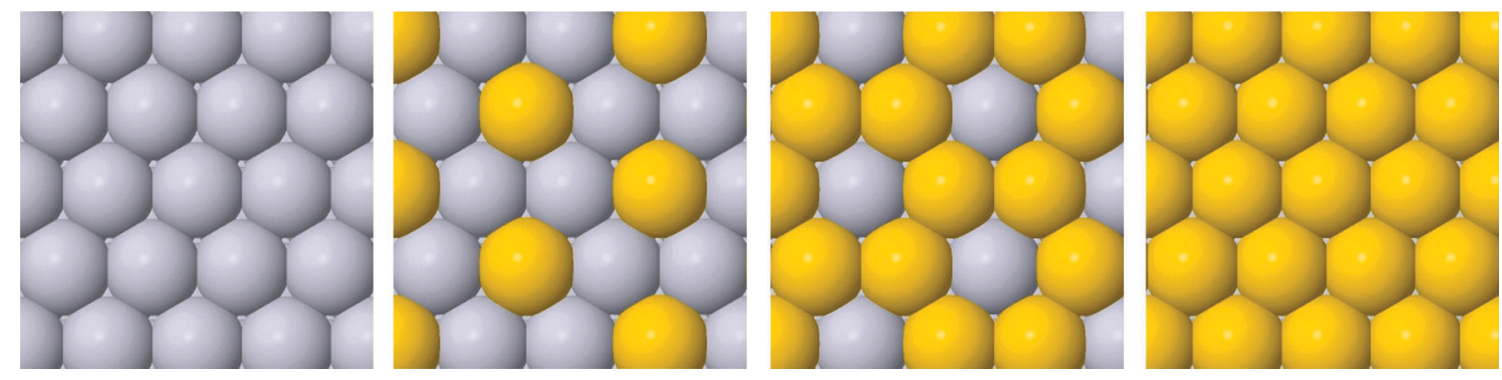

Fig. 1 Four possible atom arrangements of solute and host elements in the surface layer. 
a)

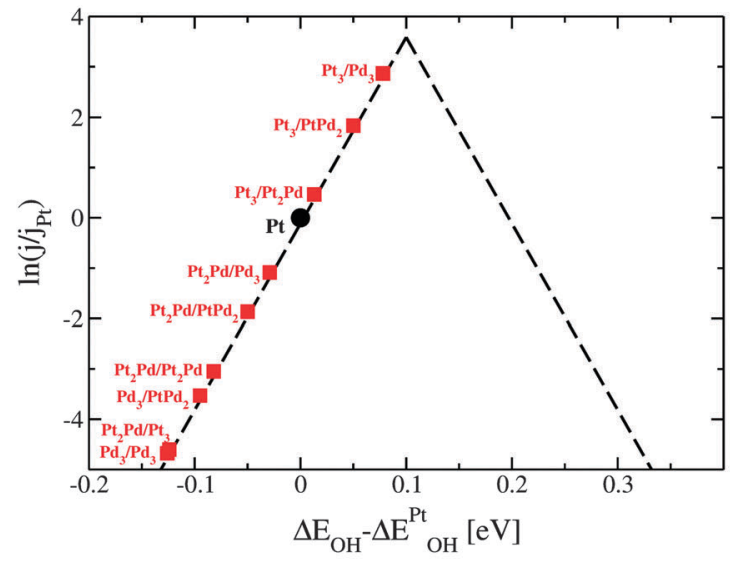

c)

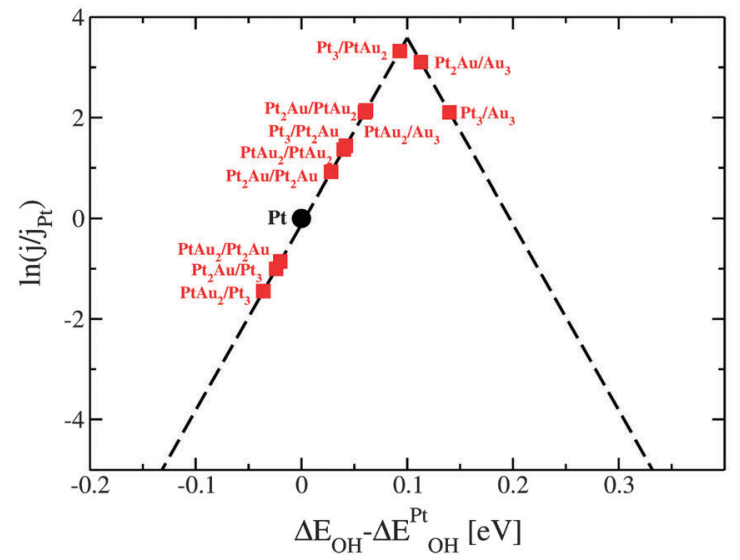

b)

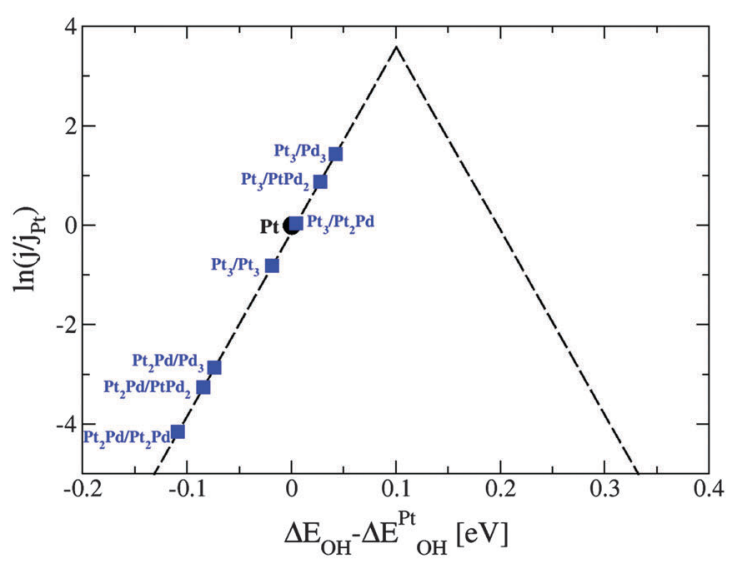

d)

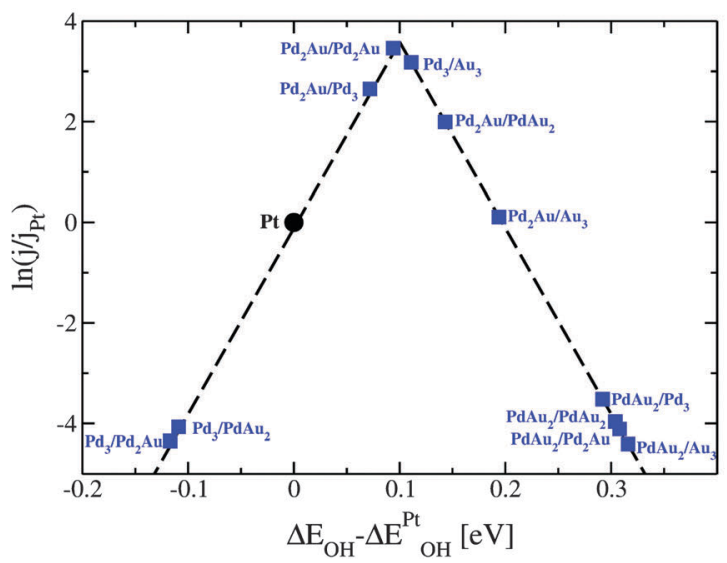

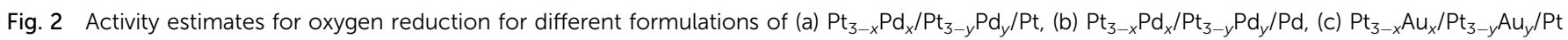
and (d) $\mathrm{Pd}_{3-x} \mathrm{Au}_{x} / \mathrm{Pd}_{3-y} \mathrm{Au}_{y} / \mathrm{Pd}$ thin films. To span the full composition space the number of atoms, $x$ and $y$, changes independently by increments of 1 . The points are color-coded depending on the host metal element; those with Pt are red and Pd blue.

The BEs of ORR intermediates are mutually correlated through the so-called linear scaling relations. ${ }^{43,44}$ In a theoretical view, the ORR activity is determined by the largest thermochemical free energy barrier between any two consecutive steps, and can therefore be approximated by the differences in the binding free energies of the reaction intermediates assigned to each electrochemical step. ${ }^{45}$ Hence, by utilizing the linear scaling relations, one can get an activity estimation plot based only on the binding energy of a single intermediate. ${ }^{43-45}$ The activity estimation plot is commonly known as a volcano plot. The volcano plot is delimited by potentials at which the first electrochemical step (right volcano leg), $\mathrm{O}_{2}$ reduction to $\mathrm{OOH}$, and the last electrochemical step (left volcano leg), $\mathrm{OH}$ reduction to water are in equilibrium, that is, equal in free energy. The activities of the binary alloys are presented on the volcano plots in Fig. 2 using the $\mathrm{OH} \mathrm{BE}$ as activity descriptor. The $\mathrm{OH}$ BEs are calculated with reference to $\mathrm{H}_{2} \mathrm{O}$ and $\mathrm{H}_{2}$ using calculated zero-point energies and tabulated values for entropies (see ref. 45 for more details).

Almost all thin films with the activities greater than that of Pt have at least $1 / 3 \mathrm{ML}$ solute element content in the subsurface layer. The weakening of the $\mathrm{OH} \mathrm{BE}$ is explained through a ligand effect brought about by the subsurface solute atoms. The influence of the ligand effect depends on the host and solute elements. It is weak for PtPd and greater for the PtAu and PdAu thin films. For thin films that have a high $\mathrm{Au}$ concentration on the surface, and are predicted to be very active for the ORR (e.g. $\mathrm{PtAu}_{2} / \mathrm{Au}_{3} / \mathrm{Pt}$ ), the 4-electron reduction to water will take place through a non-surface mediated splitting of the $\mathrm{OOH}$ bond. ${ }^{46,47}$ The strain effect is fixed and given by the metal host. Since Pt and Pd have very similar lattice constants, the strain imposed on the surface is similar. The influence of the strain effect can be best discerned by comparing data in Fig. $2 a$ and $b$.

The ligand effect of $\mathrm{Au}$ is at first glance counterintuitive. Pt or $\mathrm{Pd}$ overlayers bind more weakly in the presence of subsurface $\mathrm{Au}$, suggesting that the $\mathrm{OH}$ BE should be stronger on these alloys. The reason why the opposite is seen is because the average number of electrons in the surface layer, subsurface layer and $\mathrm{OH}$ is further away from fulfilling the octant rule in comparison to $\mathrm{OH}$ on pure $\mathrm{Pt}$ or $\mathrm{Pd}^{48}$

2.1.2. Stability. Besides the activity, the stability is the most important requirement for making a good ORR catalyst. Pt based catalysts suffer greatly from activity loss caused by particle sintering 
Table 1 Bulk formation energies in eVs specified on a per atom basis. The values left of the (/) sign are for relaxed unit cells and on the right for unit cells fixed to the Pt/Pd lattice constant. The values with the minus (-) sign are thermochemically favored

$\begin{array}{llllll}\mathrm{PtAu}_{3} & 0.035 / 0.172 & \mathrm{PdAu}_{3} & -0.072 / 0.068 & \mathrm{PtPd}_{3} & -0.004 /-0.003\end{array}$ $\begin{array}{llllll}\mathrm{Pt}_{2} \mathrm{Au}_{2} & 0.080 / 0.143 & \mathrm{Pd}_{2} \mathrm{Au}_{2} & -0.072 /-0.016 & \mathrm{Pt}_{2} \mathrm{Pd}_{2} & -0.013 /-0.012\end{array}$ $\begin{array}{llllll}\mathrm{Pt}_{3} \mathrm{Au} & 0.054 / 0.071 & \mathrm{Pd}_{3} \mathrm{Au} & -0.035 /-0.019 & \mathrm{PtPd}_{3} & -0.014 /-0.014\end{array}$

and/or dissolution under a harsh cathode environment. ${ }^{2}$ Here, we show that the stabilities of the binary thin films are strongly correlated to their composition. Once an alloy is formed, its composition is dictated by the stability under reaction conditions. In the following, we examine the stability-composition relationships by exploring (1) bulk stability, (2) surface segregation, (3) adsorbate-induced surface segregation, (4) site preference for diffusion and (5) organization of solute atoms at the surface.

We start the stability analysis by discussing the calculated bulk alloy formation energies in Table 1 . We assumed that the alloys crystallize in the face-centered-cubic crystal structure, given that this is the native crystal structure of all the metals. Formation energies are a first indication of how facile is to retain different compositions. Owing to the low alloying energies, the noble metal alloys form solid solutions with no distinct atom arrangements as in the case of e.g. intermetallic alloys. The activity of these catalysts is given by a weighted sum of activities over a range of possible compositions. As expected, the formation energies are lower when the unit cell is allowed to relax than when it is constrained to the $\mathrm{Pt} / \mathrm{Pd}$ lattice, with the differences increasing with the solute element content and generally being higher for $\mathrm{Au}$ containing alloys. The latter values are more relevant because the surface unit cell of the thin films is fixed to the Pt/Pd lattice constant.

The change in composition can happen either between different layers or within the same layer. In the former case, the change is known as surface segregation and in latter case surface diffusion. Surface segregation can be further separated into an intrinsic surface segregation (ISS) and an adsorbate-induced surface segregation (AISS) depending on a driving force. For the ISS, the main driving force is the difference in surface energies between the elements in the alloy. The driving force for the AISS is the difference in the BE of adsorbate on different elements forming the alloy. The ISS and OH/O AISS are schematically illustrated in Fig. 3 on the example of the $\mathrm{Pt}_{3} / \mathrm{Au}_{3} / \mathrm{Pt}$ near surface thin film.

It is important to note that the OH/O AISS energies also include the ISS energy of the second component. Applied to the above example, in order to get the OH/O AISS energy of Pt, one has to subtract the ISS of Au from the OH/O AISS energy of the total system. This is because Pt segregates to the surface simultaneously with Au displacement to the subsurface layer (see Fig. $3 \mathrm{~b}$ and c). Hereafter, the AISS of the second component is simply termed the AISS. The ISS and OH/O AISS energies at 1/3 ML coverage are shown in Fig. 4 for the bimetallic thin films as a function of the number of solute atoms in the subsurface layer. The reported energies are integral energies referring to a simultaneous exchange of all solute atoms between the subsurface and surface layer. The corresponding energies for substitution between the 2 nd and the 3rd layer are typically more than an order of magnitude smaller. This indicates that there is a very strong driving force for surface segregation from subsurface and a small driving force for segregation beneath the surface layers. Actually, it has been shown that Au deep inside nanoparticles can prevent dissolution of a non-noble component in multimetallic Pt alloys. ${ }^{49,50}$ Which energy prevails, the ISS or AISS energy, is given by differences between the red and blue/ green lines in Fig. 4. The two segregation energies for the PtPd thin films are almost identical owing to similar surface energies of the two metals. Pd displays a slight preference for the surface over the bulk, which is in agreement with experimental surface energies of the two metals. ${ }^{51}$ For the PtAu and PdAu thin films, the ISS energies of Au are generally very high. They are highest for the 1/3 ML Au subsurface concentration and decay monotonically per $\mathrm{Au}$ atom with increasing $\mathrm{Au}$ content. Au has a somewhat higher propensity to segregate to the surface in PtAu than PdAu thin films. Moreover, the OH/O AISS energies are higher on PdAu than PtAu, implying that Au segregation will more readily occur in PtAu than PdAu thin films. The energy gain from Au segregation can be offset by a potential dependent b)

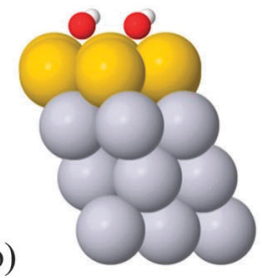

a)
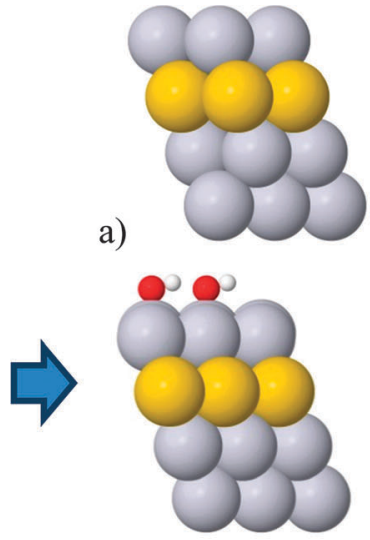
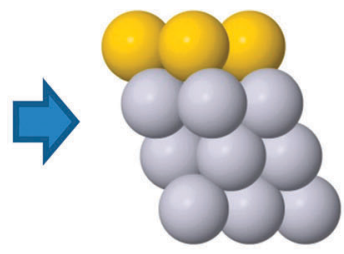

c)
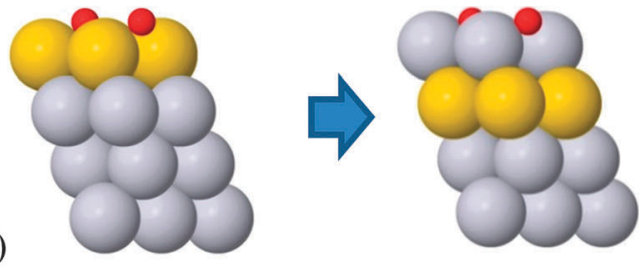

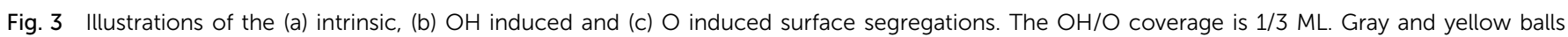
represent $\mathrm{Pt}$ and $\mathrm{Au}$ atoms, respectively. 


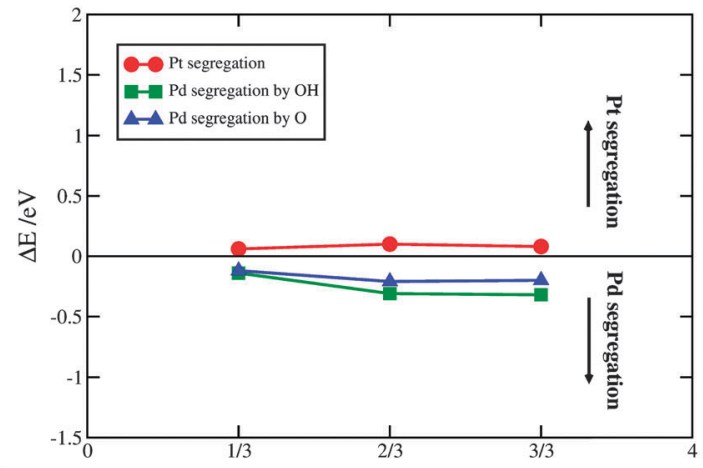

a)

ML Pd

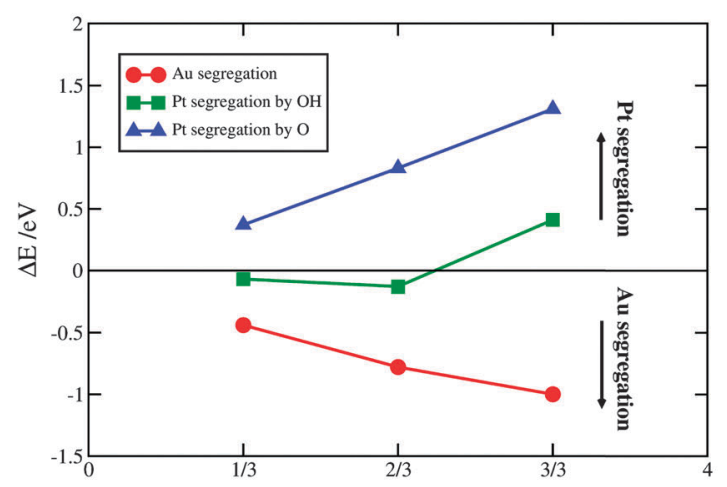

b)

$\mathrm{ML} \mathrm{Au}$

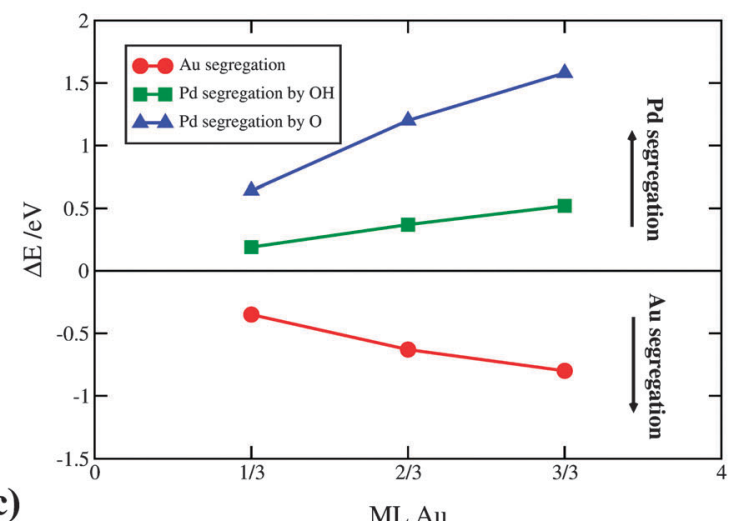

Fig. 4 Integral surface and $\mathrm{OH} / \mathrm{O}$ induced segregation energies on the (a) PtPd, (b) PtAu and (c) PdAu thin films as a function of the number of solute atoms in the subsurface layer.

$\mathrm{O}$ induced platinum segregation. The resulting direction of segregation depends on the coverage of oxygenated species. It is clear from Fig. 4 that the $1 / 3 \mathrm{ML} \mathrm{OH}$ coverage is not sufficient to instigate $\mathrm{Pt}$ segregation. This is achieved only if $\mathrm{OH}$ is entirely oxidized to $\mathrm{O}$. It should be noted that running reactions at potentials corresponding to $1 / 3 \mathrm{ML} \mathrm{O}$, i.e. $1.0 \mathrm{~V}$, is not possible because kinetic limitations set in already at potentials corresponding to $c a$. 1/3 ML $\mathrm{OH}$ coverage, i.e. $0.85 \mathrm{~V}^{52,53}$ Avoiding activity losses caused by $\mathrm{Au}$ segregation can be accomplished by periodical potential excursions to higher potentials. In reality, this happens during stop-and-go drives, where the potential occasionally jumps to $1.5 \mathrm{~V}$.

2.1.3. Site preference. So far, we have only discussed changes in the composition caused by segregation. As stated earlier, the changes can also occur within the same layer. These changes include diffusion of solute atoms to surface defects and/or selfassembling to form surface alloys or larger surface aggregates.

Solute atoms that segregate to the surface can organize in different ways, e.g. forming a uniform distribution, such as a surface alloy or homogenous islands. The outcome is given by the preference of solutes to be surrounded by other solute atoms or host element atoms. To elucidate the surface ordering of solutes, we have performed calculations with fixed $1 / 3$ and $1 / 6$ ML surface concentrations of solutes in an otherwise pure Pt or Pd host. Here, we employed a larger non-orthogonal (111) cell with $3 \times 3$ surface atoms. The energy differences between two structure extremities are shown in Fig. 5.

As seen, there is a tiny driving force for making Pd rich domains in Pt and a larger driving force for making Au rich domains in Pt. Au on the $\operatorname{Pd}(111)$ surface, on the other hand, energetically prefers to form surface alloys. The higher the solute concentration, the lower the probability the solute atoms will form islands. In the following, we discuss how surface ordering of solute atoms affects the ORR activity. For Pt with $2 / 3 \mathrm{ML}$ surface $\mathrm{Au}$ the activity reduces by $0.08 \mathrm{eV}$ (more than an order of magnitude) going from the uniform to energetically more stable island distribution. For the lower, 1/3 ML Au concentration, the effect on the activity is negligible. For the PdAu thin films the $\mathrm{OH}$ $\mathrm{BE}$ increases (becomes more negative) when surface Au atoms organize into islands irrespective of the solute element content. This is detrimental for the activity, however, as the island formation is not favored in the PdAu thin films, their activities will not be appreciably affected. Formation of uniform $\mathrm{Pd}-\mathrm{Au}$ distributions is very good from a perspective of the active $\mathrm{Pd}_{2} \mathrm{Au} / \mathrm{Pd}_{3}$ thin film. The theoretically estimated activity is more accurate because the assumed distribution model is uniform (see Fig. 1).

2.1.4. Coordination preference. In order to elucidate the site coordination preference for surface segregation, we have modeled the diffusion of solute atoms on a stepped surface of the host metal. The selected, stepped (221) surface has 4 atomswide terraces terminated by $(111) \times(111)$ steps (see illustrations in Fig. 6). The terrace width is sufficiently large to construct diffusion energy profiles from a terrace to a step site. The energy profiles are presented in Fig. 6 as a function of the distance from the step. For simplicity, the analysis is limited to a low solute atom surface concentration corresponding to $1 / 8 \mathrm{ML}$. Although, considering a particular concentration is insufficient for a comprehensive analysis, it can point to a general tendency for diffusion. We assume that the binding on a third atomic row from the step is the same as on the defect-free (111) terraces. For the adsorbate-mediated diffusion, the $\mathrm{OH} / \mathrm{O}$ coverage is fixed to $1 / 2 \mathrm{ML}$ with respect to the number of step sites $(1 / 8 \mathrm{ML}$ if the terrace atoms are also accounted for). The most favorable $\mathrm{O}$ binding configuration is in a three-fold hollow site behind the step on both $\mathrm{Pt}(221)$ and $\mathrm{Pd}(221)$. For $\mathrm{OH}$, the most stable adsorption configuration with co-adsorbed water is on a top site. Co-adsorbed water has not been explicitly modelled, which is equivalent to assuming that the effect of water on $\mathrm{OH}$ is not affected by the nature of the step atom and its surroundings. 


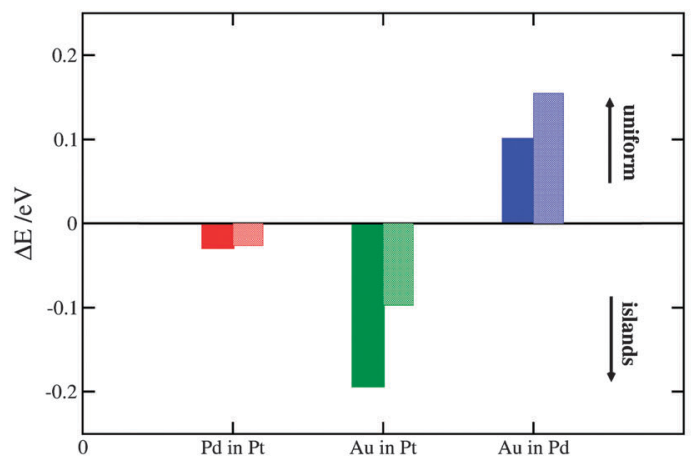

\begin{tabular}{|l|c|c|}
\hline $\begin{array}{l}\text { Surface } \\
\text { composition }\end{array}$ & Arrangement & $\begin{array}{l}\mathrm{BE}(\mathrm{OH}) \\
\text { vs. Pt }\end{array}$ \\
\hline $\mathrm{Pt}_{2} \mathrm{Au}$ & uniform & -0.023 \\
\hline $\mathrm{Pt}_{2} \mathrm{Au}$ & islands & -0.027 \\
\hline $\mathrm{PtAu}_{2}$ & uniform & -0.036 \\
\hline $\mathrm{PtAu}_{2}$ & islands & -0.116 \\
\hline $\mathrm{Pd}_{2} \mathrm{Au}$ & uniform & 0.072 \\
\hline $\mathrm{Pd}_{2} \mathrm{Au}$ & islands & -0.118 \\
\hline $\mathrm{PdAu}_{2}$ & uniform & 0.072 \\
\hline $\mathrm{PdAu}_{2}$ & islands & -0.236 \\
\hline
\end{tabular}
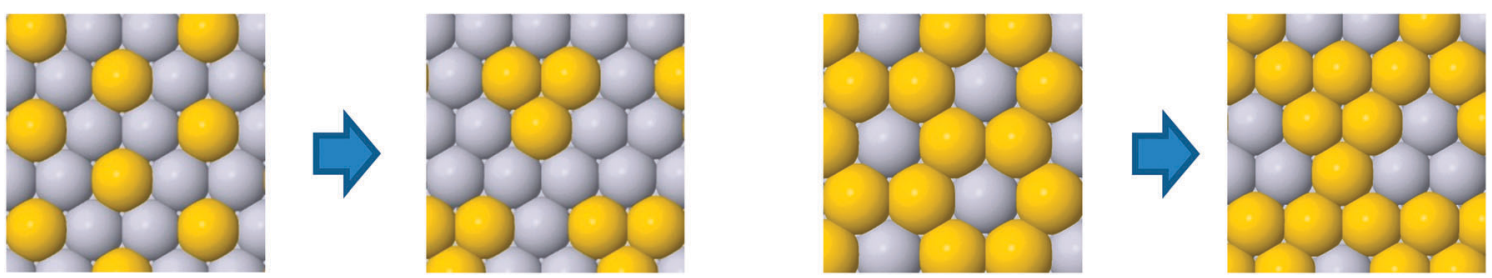

Fig. 5 The plot shows the energy difference between the uniform and cluster arrangement of solute atoms in the surface layer as illustrated below. Solid and transparent columns are for $1 / 3 \mathrm{ML}$ and $2 / 3 \mathrm{ML}$ surface solute concentration, respectively. The tabulated values show the changes in the $\mathrm{OH} B E$ for the two extremes for PtAu and PdAu thin films. Values are given in eVs. The reference value is $+0.1 \mathrm{eV}$ relative to Pt that corresponds to the OH BE at the top of the volcano.

For Pd on Pt(221), the energy differences are small, which supports the notion that alloys of Pt with Pd are typical solid solutions of the two metals. For diffusion of $\mathrm{Au}$ on $\mathrm{Pt}(221)$ and $\operatorname{Pd}(221)$ there is a significant difference that favors Au segregation to the step. This difference can be rationalized through a higher surface energy gain from moving an Au atom from a terrace to a step site than a Pt atom in the opposite direction. Here, we have used the stepped crystal as the simplest model for a surface defect. We expect that an even larger energy gain is obtained on more under-coordinated sites. Diffusion of $\mathrm{Au}$ to defects can add interpretation to experimental evidences in which small amounts of $\mathrm{Au}$ are found to stabilize Pt nanoparticles against dissolution. The results further evince that the formation of oxygen on defects, which sets in at lower potentials than on terraces,${ }^{54}$ will force $\mathrm{Au}$ atoms to change the direction of diffusion, from steps to terraces.

2.1.5. Activity versus stability. The $\mathrm{OH} \mathrm{BE}$ is used as activity descriptor to estimate the ORR activity. As stability descriptor we choose the surface energy. Surface energies of the bimetallic thin films are calculated through eqn (1) and (2).

$$
\begin{gathered}
E_{\mathrm{A}_{m} \mathrm{~B}_{n}}^{\text {surf }}=E_{\mathrm{A}_{m} \mathrm{~B}_{n}}-m E_{\mathrm{A}}^{\text {bulk }}-n E_{\mathrm{B}}^{\text {bulk }}-E_{\mathrm{B}}^{\text {surf }} \\
E_{\mathrm{B}}^{\text {surf }}=\left(E_{(m+n) \mathrm{B}}-(m+n) E_{\mathrm{B}}^{\text {surf }}\right) / 2
\end{gathered}
$$

Here, we chose a different $A_{m} B_{\mathrm{n}}$ notation because the total number of atoms A and B in the unit cell becomes important. This notation is used only at this time to explain the approach for obtaining surface energies; for distinguishing among the different thin films we keep the former notation, introduced in Section 2.1. $E_{\mathrm{A}_{m} \mathrm{~B}_{n}}^{\text {surf }}$ and $E_{\mathrm{B}}^{\text {surf }}$ are surface energies of an $\mathrm{A}_{m} \mathrm{~B}_{n}$ binary thin film (A-solute and B-host element) and B, respectively, where $m$ and $n$ are the total numbers of $\mathrm{A}$ and $\mathrm{B}$ atoms in the slab. For the $\sqrt{ } 3 \times \sqrt{ } 3 R 30^{\circ}$ surface unit cell and 4 layers thick slabs, the number of atoms, $m+n$, in the slab sums up to $12 . E_{\mathrm{B}}^{\text {bulk }}$ and $E_{\mathrm{A}}^{\text {bulk }}$ are the energies of A and B in bulk and $E_{(m+n) \mathrm{B}}$ is the total energy of the fixed slab made of pure element $B$. The first three terms in eqn (1) are used to calculate surface energies of $A_{m} B_{n}$ thin films. As each slab has two surfaces exposed to vacuum, in order to get the surface energy of a thin film terminating side of the slab one has to subtract the surface energy of metal B, $E_{\mathrm{B}}^{\text {surf }}$, from backside of the slab. Eqn (2) is used to estimate $E_{\mathrm{B}}^{\text {surf }}$ using a similar rationale as for $\mathrm{A}_{m} \mathrm{~B}_{n}$. Number 2 enters into eqn (2) because slab B has two equivalent surfaces. Here, we have used the bulk values as references, which is justified by the small alloying energies of the thin films (see Table 1). If the alloy formation energies were larger then the chemical potential of the solute element in the alloy would be the right reference.

To select the best bimetallic thin films catalysts, in Fig. 7 we plot the activity against the stability. The activity is approximated by the $\mathrm{OH} \mathrm{BE}$ on the $x$-axis and the stability by surface energy on the $y$-axis. The grey box in Fig. 5 designates the area, where the activities are higher than that on pristine $\mathrm{Pt}(111)$; that is, for the $\mathrm{OH} \mathrm{BE}$ values ranging from that on $\mathrm{Pt}(111)$ to a symmetric point on the opposite side of the volcano. As seen, for the binary PtPd systems (red triangles) it is best to have the near-surface alloy of Pd in Pt or Pt skin on Pd (see the ESI $\dagger$ ). 
a)

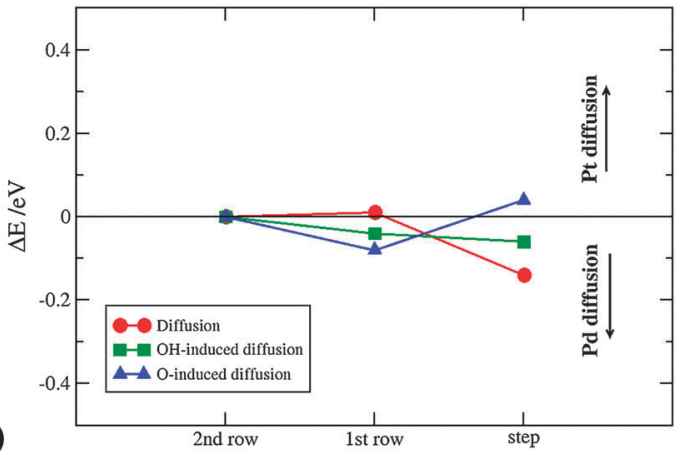

b)

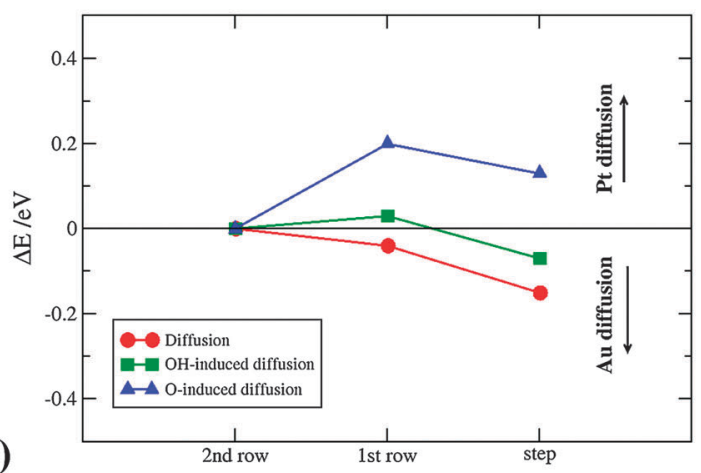

c)
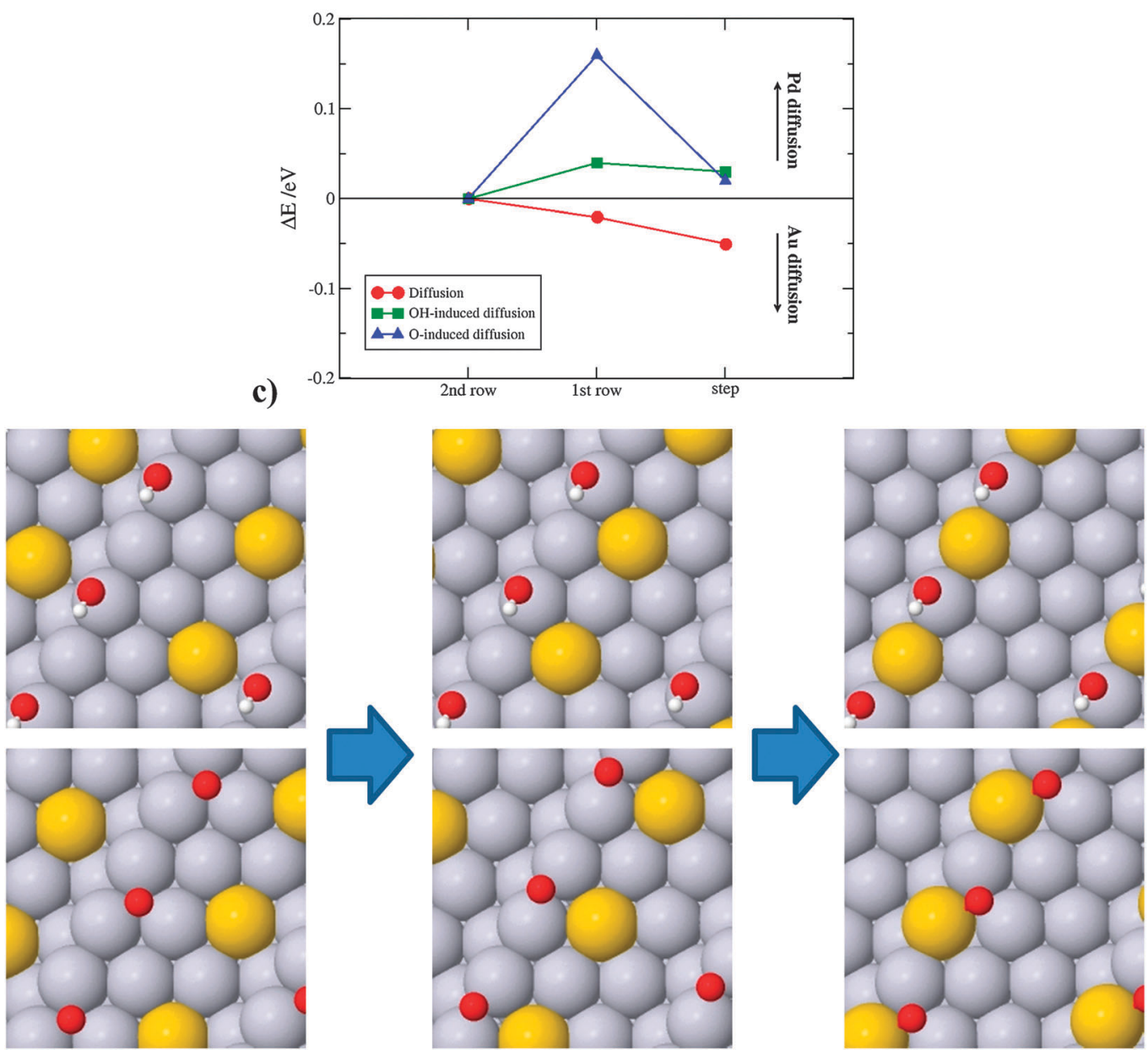

Fig. 6 Diffusion energy profiles from terraces to steps for a surface (a) Pd atom on Pt(221) (b) Au atom on Pt(221) and (c) Au atom on Pd(221). The illustrations show top views for diffusion of a Au atom on the Pt(211) surface. 


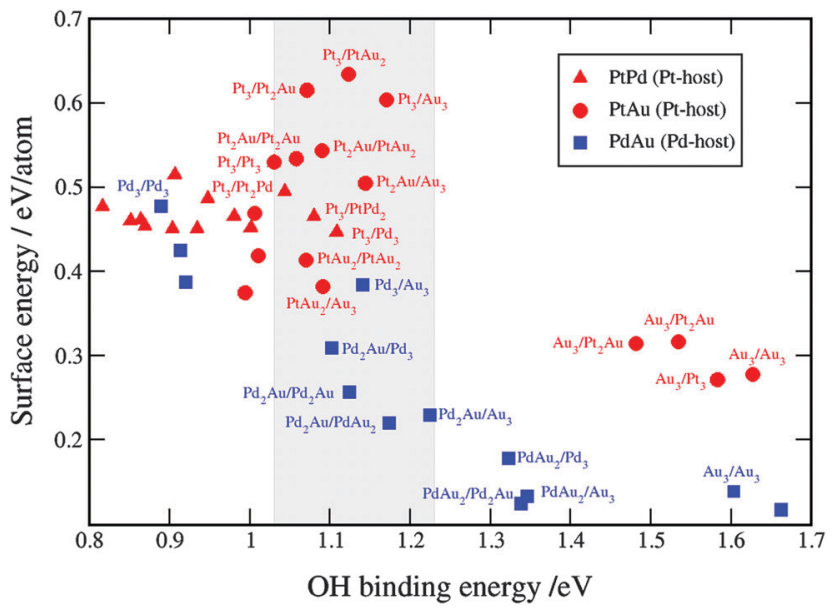

Fig. 7 Comparison between the activity and stability descriptors used to assess the catalyst performance. The grey shaded box is the area with the activities higher than that on $\mathrm{Pt}(111)$. For clarity reasons, only points with the $\mathrm{OH}$ binding energies higher than that on Pt are labeled. A table with the surface and $\mathrm{OH}$ binding energies for the bimetallic systems is given in note 3 in the ESI. $\dagger$

Notice that surface energies for the PtPd thin films change linearly with the Pd content in the subsurface layer. There are many PtAu and PdAu thin film formulations that fall in the designated area, however, many of these are not stable owing to high surface energies. Even those formulations with $\mathrm{Au}$ that have low surface energies might be considered unstable because if $\mathrm{Au}$ is present in excess, it will segregate to the surface and change the thin film compositions to those with smaller surface energies and lower activities for the ORR (see the points in the lower-right corner of Fig. 7). However, if the amount of $\mathrm{Au}$ is carefully dosed, e.g. in $\mathrm{Pd}$ to form $\mathrm{Pd}_{2} \mathrm{Au} / \mathrm{Pd}_{3}$, then the $\mathrm{Pd}_{2} \mathrm{Au} / \mathrm{Pd}_{3}$ thin film might be a good catalyst for the ORR. It is noteworthy that surface energies of the PdAu thin films are generally lower than those for the PtAu or PtPd thin films. This is a result of several effects: (1) the computed Pd surface energy is lower than Pt surface energy, (2) the formation energies of PdAu alloys are greater than those of PtAu alloys (see Table 1) and (3) the assumed distribution is uniform, which is energetically the less stable configuration for the PtAu thin films (see Fig. 5).

\subsection{Ternary thin films}

2.2.1. Activity. In addition to the binary thin films, we have also modeled the activity of ternary Pt, Pd, Au thin films for oxygen reduction. Eight different ternary systems are examined in total, four with pure metal skins (two with Pt and two with Pd host) and four with mixed Pt-Pd skins. Thin films with Pt host include near-surface alloys of PdAu in Pt, Pd skins on Pt with subsurface $\mathrm{Au}$ and mixed Pt-Pd skins with some additional $\mathrm{Au}$ and/or Pd in the subsurface layer. Ternary alloys with Pd host are obtained by substituting Pt with Pd and Pd with Pt in the thin films with Pt host. Activity estimates for the selected ternary thin films are shown in Fig. 8. For clarity, the results are divided into two plots depending on the composition of the surface layer. Data for the pure Pt-Pd skins are shown in Fig. 8a and for the mixed metal skins in Fig. 8b. There are many formulations with both surface and subsurface Au that have the activities higher than that of Pt, especially in the case of the pure metal skins.

2.2.2. Activity $\boldsymbol{v s}$. stability. To select the best ternary thin films we plot the surface energy as a function of the $\mathrm{OH} \mathrm{BE}$ in Fig. 9. Again, we make use of eqn (1) and (2) with the difference that eqn (1) will have an additional term for the third component. As expected, the results are similar to those for bimetallic thin films with Au. Owing to a much lower surface energy than either Pt or Pd, Au energetically always prefers to be on the surface. Thus, active formulations with subsurface $\mathrm{Au}$ will gradually become deactivated due to surface Au segregation. Nevertheless, there are some formulations with surface $\mathrm{Au}$, $\mathrm{Pt}_{2} \mathrm{Au} / \mathrm{Pd}_{3} / \mathrm{Pd}$ and $\mathrm{PtAu}_{2} / \mathrm{Pd}_{3} / \mathrm{Pd}$ that have reasonably low surface energies and are active for the ORR. We conclude that the best ternary catalysts are Pt skins on Pd with a small amount of $\mathrm{Au}$ whose coverage after surface segregation should not exceed 2/3 ML. Small amounts of surface Au will as well retard platinum dissolution through a charge donation to vicinal undercoordinated surface Pt atoms.
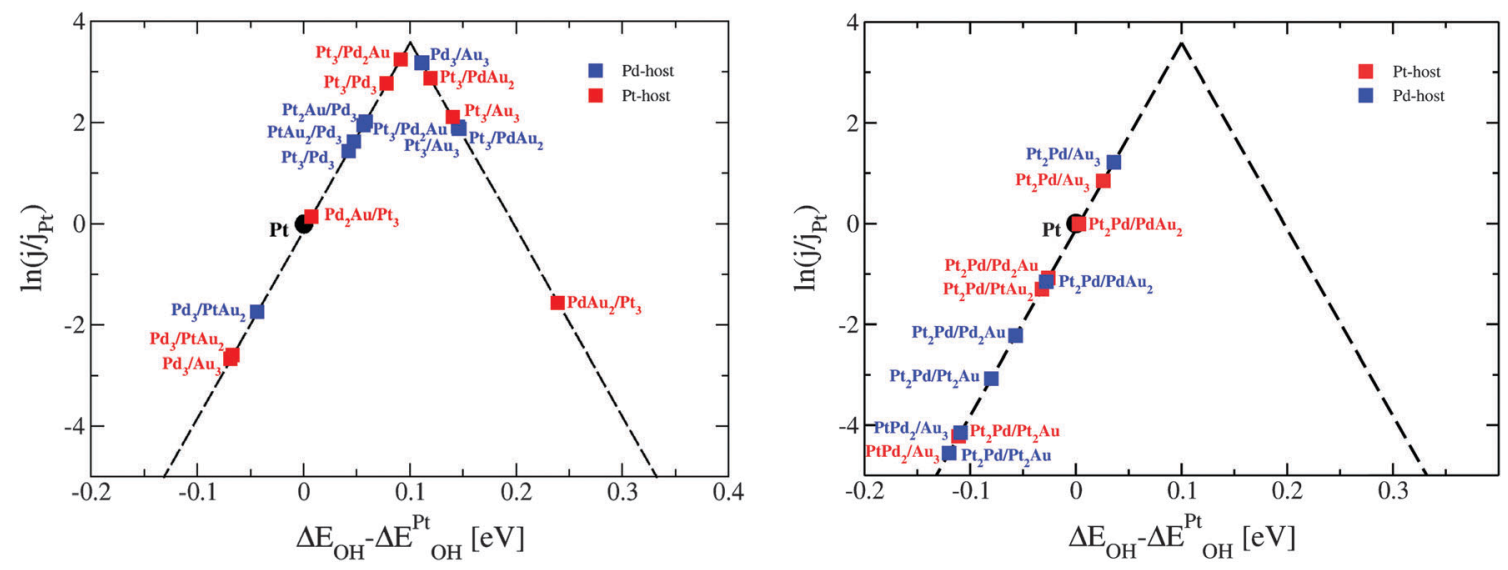

Fig. 8 Activity estimates for oxygen reduction of ternary thin film alloy surfaces with (a) pure Pt or Pd skin and (b) mixed Pt-Pd skin. 


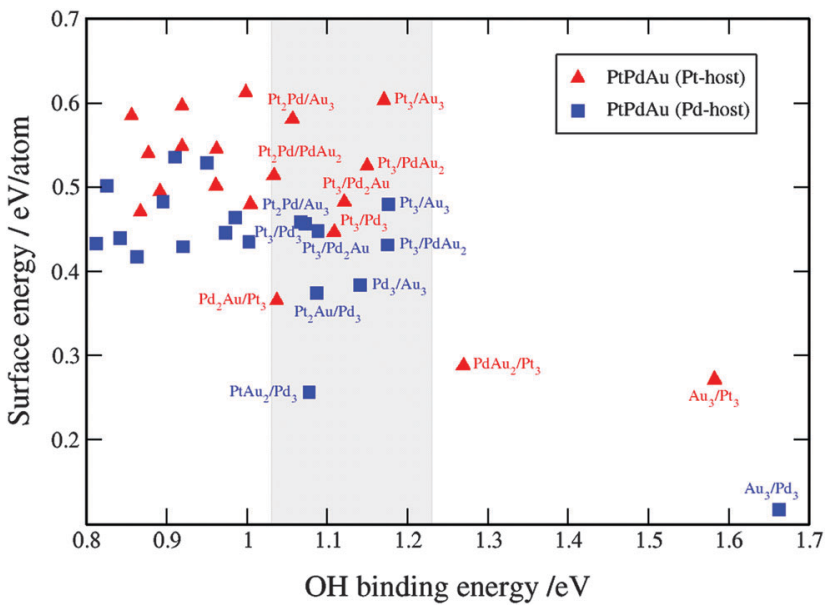

Fig. 9 Comparison between the activity and stability descriptors used to assess the catalyst performance. The grey shaded box is the area with the activities higher than that of $\mathrm{Pt}(111)$. For clarity reasons, only points with the $\mathrm{OH}$ binding energies higher than that of Pt are labeled. A table with the surface and $\mathrm{OH}$ binding energies for the ternary systems is given in note 3 in the ESI.

\section{Conclusions}

In summary, we have shown that certain binary and ternary Pt, $\mathrm{Pd}$ and $\mathrm{Au}$ thin films display high activities towards the ORR. The major conclusions can be summarized in the following points.

(1) The activity is very sensitive to the alloy composition. Due to the low alloy formation energies the thin films form solid solutions with a range of compositions. It might be therefore, difficult to retain the composition with the highest activity. Nonetheless, in the case of many thin films, there are several different formulations with high activities. For instance, as long as Pd in the binary PtPd thin films is restricted below the surface layer the activities will remain high. Moreover, the higher the Pd subsurface content the more stable the alloy. As for the binary and ternary thin films with $\mathrm{Au}$, there are many formulations with $\mathrm{Au}$ in the surface and/or subsurface layer that are quite active for oxygen reduction. However only few of these are stable, such as $\mathrm{Pd}_{2} \mathrm{Au} / \mathrm{Pd}_{3} / \mathrm{Pd}$ or $\mathrm{PtAu}_{2} / \mathrm{Pd}_{3} / \mathrm{Pd}$, and that is under condition that there is no excess Au that can change the compositions over time towards more Au enriched surfaces.

(2) Solute elements in the subsurface layer are generally found to weaken the $\mathrm{OH} \mathrm{BE}$ through the ligand effect. The higher the solute atom concentration, the more the $\mathrm{OH} \mathrm{BE}$ is weakened. Having Pt or Pd in the bulk (below the subsurface layer) does not appreciably affect the results (see Fig. 2a and b for comparison). Strain effects are essentially the same for the two metal hosts, whilst ligand effects from the 3rd and subsequent layers are largely screened at the surface.

(3) There is a large thermochemical driving force for $\mathrm{Au}$ to segregate to the surface. The segregation energy can be offset by oxygen mediated $\mathrm{Pt} / \mathrm{Pd}$ segregation. This will happen only if the oxygen coverage on the surface is $1 / 3 \mathrm{ML}$ or greater. As this coverage is attained at potentials at which the ORR is kinetically inhibited, occasional excursions to higher potentials are required to recover the activity, i.e. the lost platinum surface area.

(4) There is a thermochemical driving force for $\mathrm{Au}$, once segregated to the surface, to diffuse to more under-coordinated sites. Au renders Pt less prone to dissolution through charge transfer effects. Diffusion of Au atoms to defects can explain high stabilities observed for Pt nanoparticles decorated by $\mathrm{Au}$ clusters. In addition, Au in Pt shows a tendency to self-assemble into islands and in Pd into surface alloys.

(5) There is almost no, or a very little, driving force for Pd to segregate to the Pt surface irrespective of the surface oxidation state. In addition, Pd in Pt shows no preference for defects or surface self-assembling.

On the basis of the presented data, we conclude that the binary PtPd thin films, in which Pt comprises the skin and Pd is restricted to the subsurface layer(s) are the best catalysts for oxygen reduction. Addition of a small, controlled amount of $\mathrm{Au}$ can, however, further improve the performance of the PtPdAu thin films (e.g. $\mathrm{Pt}_{2} \mathrm{Au} / \mathrm{Pd}_{3} / \mathrm{Pd}$ and $\mathrm{PtAu}_{2} / \mathrm{Pd}_{3} / \mathrm{Pd}$ ) by retarding the dissolution of under-coordinated $\mathrm{Pt}$ atoms, and perhaps even enhancing the activity, making the ternary PtPdAu systems interesting for the ORR in PEMFCs.

\section{Theory}

Total energies are calculated using density functional theory (DFT) calculations employing grid-based projector-augmented wave method (GPAW) code integrated with atomic simulation environment (ASE). ${ }^{55}$ Calculations are performed using the RPBE exchange-correlation functional ${ }^{56}$ and a grid spacing of $0.15 \AA$ A. The RPBE relaxed lattice constants of Pt and Pd are 3.991 and $3.980 \AA$, respectively. The occupation of the one-electron states is calculated at an electronic temperature of $k_{\mathrm{B}} T=0.1 \mathrm{eV}$, and then extrapolated to $k_{\mathrm{B}} T=0 \mathrm{~K}$.

$\mathrm{Pt}(111)$ and $\mathrm{Pt}(221)$ electrodes are represented by periodically repeated slabs separated by at least $10 \AA$ vacuum. Two unit cells with $(\sqrt{ } 3 \times \sqrt{ } 3) R 30^{\circ}$ and $(3 \times 3) R 30^{\circ}$ size in the surface plane are used to represent the (111) surface. The $\operatorname{Pt}(221)$ electrode was made from the $2 \times 4 \mathrm{Pt}(111)$ slab by adjusting the unit cell parameters. The slab thickness for all the electrodes is 4 layers. The bottom two layers are fixed both in terms of composition and coordinates, while the remaining atoms and adsorbates are allowed to relax in order to assume minimum energy positions. In the ESI $\dagger$ we also provide a table showing how the BEs relative to $\mathrm{Pt}(111)$ change as a function of the number of slab layers.

The total number of configurations for each binary system given the unit cell size is $4^{2}$. For all surfaces the Brillouin zone was sampled by a $4 \times 4 \times 1$ Monkhorst-Pack $k$-point grid, which was sufficient to obtain converged results. ${ }^{57}$ The convergence is reached when the sum of absolute forces acting on the atoms becomes less than $0.05 \mathrm{eV} \AA^{-1}$. Symmetry and dipole corrections are applied to reduce the number of $k$-points in the Brillouin zone and to electrostatically decouple the interaction of neighboring slabs. 


\section{Acknowledgements}

The financial support of the European Commission under the FP7 Fuel Cells and Hydrogen Joint Technology Initiative grant agreement FP7-2012-JTI-FCH-325327 for the SMARTcat project and FP7-2011-JTI-FCH-303492for the CATHcat project is gratefully acknowledged. We would also like to acknowledge Paul C. Jennings for valuable discussions.

\section{References}

1 M. K. Debe, Nature, 2012, 486, 43-51.

2 H. A. Gasteiger, S. S. Kocha, B. Sompalli and F. T. Wagner, Appl. Catal., B, 2005, 56, 9-35.

3 X. Zhao, M. Yin, L. Ma, L. Liang, C. P. Liu, J. H. Liao, T. H. Lu and W. Xing, Energy Environ. Sci., 2011, 4, 2736-2753.

4 N. Markovic, H. Gasteiger and P. N. Ross, J. Electrochem. Soc., 1997, 144, 1591-1597.

5 B. B. Blizanac, P. N. Ross and N. M. Markovic, Electrochim. Acta, 2007, 52, 2264-2271.

6 S. Kondo, M. Nakamura, N. Maki and N. Hoshi, J. Phys. Chem. C, 2009, 113, 12625-12628.

7 F. H. B. Lima, J. Zhang, M. H. Shao, K. Sasaki, M. B. Vukmirovic, E. A. Ticianelli and R. R. Adzic, J. Phys. Chem. C, 2007, 111, 404-410.

8 S. Mukerjee, S. Srinivasan, M. P. Soriaga and J. McBreen, J. Electrochem. Soc., 1995, 142, 1409-1422.

9 V. R. Stamenkovic, B. Fowler, B. S. Mun, G. F. Wang, P. N. Ross, C. A. Lucas and N. M. Markovic, Science, 2007, 315, 493-497.

10 J. Greeley, I. E. L. Stephens, A. S. Bondarenko, T. P. Johansson, H. A. Hansen, T. F. Jaramillo, J. Rossmeisl, I. Chorkendorff and J. K. Nørskov, Nat. Chem., 2009, 1, 552-556.

11 R. R. Adzic, J. Zhang, K. Sasaki, M. B. Vukmirovic, M. Shao, J. X. Wang, A. U. Nilekar, M. Mavrikakis, J. A. Valerio and F. Uribe, Top. Catal., 2007, 46, 249-262.

12 S. Koh and P. Strasser, J. Am. Chem. Soc., 2007, 129, 12624-12625.

13 S. Mukerjee and S. Srinivasan, J. Electroanal. Chem., 1993, 357, 201-224.

14 A. U. Nilekar, Y. Xu, J. Zhang, M. B. Vukmirovic, K. Sasaki, R. R. Adzic and M. Mavrikakis, Top. Catal., 2007, 46, 276-284.

15 V. Climent, N. M. Marković and P. N. Ross, J. Phys. Chem. B, 2000, 104, 3116-3120.

16 M. H. Shao, T. Huang, P. Liu, J. Zhang, K. Sasaki, M. B. Vukmirovic and R. R. Adzic, Langmuir, 2006, 22, 10409-10415.

17 W. Liu, P. Rodriguez, L. Borchardt, A. Foelske, J. Yuan, A.-K. Herrmann, D. Geiger, Z. Zheng, S. Kaskel, N. Gaponik, R. Kötz, T. J. Schmidt and A. Eychmüller, Angew. Chem., Int. Ed., 2013, 52, 9849-9852.

18 C. Koenigsmann, A. C. Santulli, K. Gong, M. B. Vukmirovic, W. Zhou, E. Sutter, S. S. Wong and R. R. Adzic, J. Am. Chem. Soc., 2011, 133, 9783-9795.

19 M. Shao, G. He, A. Peles, J. H. Odell, J. Zeng, D. Su, J. Tao, T. Yu, Y. Zhu and Y. Xia, Chem. Commun., 2013, 49, 9030-9032.
20 R. Choi, S.-I. Choi, C. H. Choi, K. M. Nam, S. I. Woo, J. T. Park and S. W. Han, Chemistry, 2013, 19, 8190-8198.

21 G. Zhang, Z.-G. Shao, W. Lu, F. Xie, H. Xiao, X. Qin and B. Yi, Appl. Catal., B, 2013, 132-133, 183-194.

22 L. Liu, G. Samjeske, S. Nagamatsu, O. Sekizawa, K. Nagasawa, S. Takao, Y. Imaizumi, T. Yamamoto, T. Uruga and Y. Iwasawa, J. Phys. Chem. C, 2012, 116, 23453-23464.

23 S. M. Alia, K. O. Jensen, B. S. Pivovar and Y. Yan, ACS Catal., 2012, 2, 858-863.

24 H. Zhang, Y. Yin, Y. Hu, C. Li, P. Wu, S. Wei and C. Cai, J. Phys. Chem. C, 2010, 114, 11861-11867.

25 J. X. Wang, H. Inada, L. Wu, Y. Zhu, Y. Choi, P. Liu, W.-P. Zhou and R. R. Adzic, J. Am. Chem. Soc., 2009, 131, 17298-17302.

26 G. V. Fortunato, L. B. Venarusso and G. Maia, ChemElectroChem, 2014, 1, 625-636.

27 B. Y. Xia, H. B. Wu, Y. Yan, X. W. D. Lou and X. Wang, J. Am. Chem. Soc., 2013, 135, 9480-9485.

28 P. Hernández-Fernández, S. Rojas, P. Ocón, J. L. Gómez de la Fuente, J. San Fabián, J. Sanza, M. A. Peña, F. J. García-García, P. Terreros and J. L. G. Fierro, J. Phys. Chem. C, 2007, 111, 2913-2923.

29 H. Lang, S. Maldonado, K. J. Stevenson and B. D. Chandler, J. Am. Chem. Soc., 2004, 126, 12949-12956.

30 J. Luo, M. M. Maye, V. Petkov, N. N. Kariuki, L. Wang, P. Njoki, D. Mott, Y. Lin and C.-J. Zhong, Chem. Mater., 2005, 17, 3086-3091.

31 C.-W. Liu, Y.-C. Wei, C.-C. Liu and K.-W. Wang, J. Mater. Chem., 2012, 22, 4641.

32 Y. Ma, H. Zhang, H. Zhong, T. Xu, H. Jin and X. Geng, Catal. Commun., 2010, 11, 434-437.

33 H.-Y. Park, T.-Y. Jeon, J. H. Jang, S. J. Yoo, K.-H. Choi, N. Jung, Y.-H. Chung, M. Ahn, Y.-H. Cho, K.-S. Lee and Y.-E. Sung, Appl. Catal., B, 2013, 129, 375-381.

34 K.-S. Lee, H.-Y. Park, H. C. Ham, S. J. Yoo, H. J. Kim, E. Cho, A. Manthiram and J. H. Jang, J. Phys. Chem. C, 2013, 117, 9164-9170.

35 J. Zhang, K. Sasaki, E. Sutter and R. R. Adzic, Science, 2007, 315, 220-222.

36 Y. Zhang, Q. Huang, Z. Zou, J. Yang, W. Vogel and H. Yang, J. Phys. Chem. C, 2010, 114, 6860-6868.

37 H. C. Ham, J. A. Stephens, G. S. Hwang, J. Han, S. W. Nam and T. H. Lim, J. Phys. Chem. Lett., 2012, 3, 566-570.

38 Y. Suo and I.-M. Hsing, Electrochim. Acta, 2011, 56, 2174-2183.

39 S.-Y. Lee, N. Jung, J. Cho, H.-Y. Park, J. Ryu, I. Jang, H.-J. Kim, E. Cho, Y.-H. Park, H. C. Ham, J. H. Jang and S. J. Yoo, ACS Catal., 2014, 4, 2402-2408.

40 J. B. Xu, T. S. Zhao, S. Y. Shen and Y. S. Li, Int. J. Hydrogen Energy, 2010, 35, 6490-6500.

41 M. Simões, S. Baranton and C. Coutanceau, Appl. Catal., B, 2010, 93, 354-362.

42 C. Koenigsmann, E. Sutter, R. R. Adzic and S. S. Wong, J. Phys. Chem. C, 2012, 116, 15297-15306.

43 J. Rossmeisl, A. Logadottir and J. K. Nørskov, Chem. Phys., 2005, 319, 178-184. 
44 F. Abild-Pedersen, J. Greeley, F. Studt, J. Rossmeisl, T. R. Munter, P. G. Moses, E. Skulason, T. Bligaard and J. K. Nørskov, Phys. Rev. Lett., 2007, 99, 16105.

45 J. K. Nørskov, J. Rossmeisl, A. Logadottir, L. Lindqvist, J. R. Kitchin, T. Bligaard and H. Jonsson, J. Phys. Chem. B, 2004, 108, 17886-17892.

46 S. Siahrostami, A. Verdaguer-Casadevall, M. Karamad, D. Deiana, P. Malacrida, B. Wickman, M. Escudero-Escribano, E. A. Paoli, R. Frydendal, T. W. Hansen, I. Chorkendorff, I. Stephens and J. Rossmeisl, Nat. Mater., 2013, 12, 1137-1143.

47 F. Calle-Vallejo, J. I. Martínez, J. M. García-Lastra, E. Abad and M. T. M. Koper, Surf. Sci., 2013, 607, 47-53.

48 F. Calle-Vallejo, J. I. Martinez, J. M. Garcia-Lastra, J. Rossmeisl and M. T. M. Koper, Phys. Rev. Lett., 2012, 108, 116103.

49 C.-E. Kim, D.-H. Lim, J. H. Jang, H. J. Kim, S. P. Yoon, J. Han, S. W. Nam, S.-A. Hong, A. Soon and H. C. Ham, J. Chem. Phys., 2015, 142, 034707.
50 Y. Kang, J. Snyder, M. Chi, D. Li, K. L. More, N. M. Markovic and V. R. Stamenkovic, Nano Lett., 2014, 14, 6361-6367.

51 L. Vitos, A. V. Ruban, H. L. Skriver and J. Kollar, Surf. Sci., 1998, 411, 186-202.

52 J. Rossmeisl, G. S. Karlberg, T. Jaramillo and J. K. Nørskov, Faraday Discuss., 2008, 140, 337-346.

53 A. S. Bondarenko, I. E. L. Stephens, H. A. Hansen, F. J. PérezAlonso, V. Tripkovic, T. P. Johansson, J. Rossmeisl, J. K. Nørskov and I. Chorkendorff, Langmuir, 2011, 27, 2058-2066.

54 V. Tripković, I. Cerri, T. Bligaard and J. Rossmeisl, Catal. Lett., 2014, 144, 380-388.

55 J. Mortensen, L. Hansen and K. Jacobsen, Phys. Rev. B: Condens. Matter Mater. Phys., 2005, 71, 035109.

56 B. Hammer, L. Hansen and J. Nørskov, Phys. Rev. B: Condens. Matter Mater. Phys., 1999, 59, 7413-7421.

57 H. J. Monkhorst, Phys. Rev. B: Condens. Matter Mater. Phys., 1976, 13, 5188. 